\title{
Terminal Velocity Maximization of Air-to-Air Missiles in Agile Turn Phase
}

\author{
Min Ki Kim ${ }^{1}$, Min-Jea Tahk ${ }^{1}$, Bong-Gyun Park ${ }^{2}$ and Yun-Young Kim² \\ ${ }^{1}$ KAIST, Department of Aerospace Engineering, 291 Daehak-ro Yuseong-gu Daejeon, Korea \\ ${ }^{2}$ LIG Nex1, PGM R\&D Laboratory, 207 Mabuk-ro Giheung-gu Yongin-si, Korea
}

\begin{abstract}
Terminal velocity maximization of air-to-air agile missiles during agile turn phase is studied. It is important to make sure that agile missiles have enough velocity after agile turn stage in order to impact the target. The agile turn is operated during the boost phase. Thus, the agile turn problem is formulated as terminal velocity maximization with given final time. Optimal solutions are obtained by two different methods, pseudospectral method and parameterized costate optimization method. The parameterized costate optimization method is a part of shooting method of indirect method which is proposed to verify the solutions obtained by pseudospectral method. At first, the basic missile model with constant axial acceleration is treated in the two dimensional yaw plane. After looking into the basic missile model, missile model with aerodynamic forces and thrust is analyzed.
\end{abstract}

\section{Introduction}

In the last few decades, advances in aircraft maneuvering technology continued to produce new challenges for antiair weapon systems. One of the challenges is to make airto-air missiles impact the target at any direction and at any aspect. This demand resulted in the development of 'agile air-to-air missiles'. The agile missiles are classified into each mission phase which includes: launch, agile turn at high angle of attack, midcourse, and endgame [1]. The agile turn problems are focused on the first two stages which are the key issues of missiles' performance because these stages affect the missiles' launch envelope. These stages are accomplished during the boost phase.

The agile air-to-air missiles have lock-on after launch ability and even can engage targets in the rear hemisphere relative to the launch aircraft. Therefore, it is important to make sure that agile missiles have enough velocity after agile turn stage in order to impact the target. Solving an optimal control problem by setting cost function as minimum agile turn time may make velocity decrease significantly. It is undesirable since it cannot use relative velocity that is earned from the launch aircraft and may results into mission failure. Thus, this paper focuses on maximum terminal velocity in the agile turn stage.

The agile turn problem has been an area of research interest for the past 20 years. However, due to heavy amount of computational workloads of obtaining the optimal solution, it has not been focused on getting the optimal trajectory. Rather, it has been examined to get over the uncertainty and nonlinearity because of high angle of attack regime during the agile turn phase [2], [3].

There have been many efforts to reduce computational workloads of obtaining the optimal solution by simplifying the missile model. Lee [4] has dealt with a nonlinear adaptive autopilot design for agile missile systems that are reduced to a two dimensional plane problem. In [4], the paper proposed a longitudinal autopilot for angle-of-attack tracking based on backstepping control methodology in conjunction with the time-delay adaptation scheme. Ryu [5] introduced the design of the angle of attack controller using the pole placement approach for the high angle of attack missiles.

This paper investigates the optimal control problem by proposing 'parameterized costate optimization method'. It is one of the shooting methods of indirect method which transforms the problem from the original optimal control problem to finding initial values of costates. By using this method to a simple missile model, solutions obtained by applying pseudospectral method [6] can be verified. Also numerical optimization is conducted on missile model with aerodynamic forces and thrust.

\section{Parameterized costate optimization method}

There are several numerical ways to solve optimal control problems. One way is using GPOPS-II software which employed an hp-adaptive version of the Radau pseudospectral method [7]. Before applying this method, it needs to be verified to apply to various complex dynamic systems. Thus, parameterized costate optimization method is proposed in this section. It is a part of shooting method of indirect method.

A final time fixed optimal control problem can be formulated as (1) and (2) with state $x(t) \in R^{n}$ and control input $u(t) \in R^{m}$ in time interval $\left[t_{0}, t_{f}\right]$. 


$$
\begin{gathered}
\dot{x}=f(x, u, t) \\
J=\phi\left(x_{f}, t_{f}\right)+\int_{t_{0}}^{t_{f}} g(x, u, t) d t
\end{gathered}
$$

where $x_{f}$ denotes final states. Approaching the optimal control problem with indirect method, the Hamiltonian function is defined as:

$$
H=g+\lambda^{T} f
$$

Then, the equations of motion can be written in Hamiltonian's form (4).

$$
\begin{aligned}
& \frac{\partial H}{\partial x}=-\dot{\lambda} \\
& \frac{\partial H}{\partial u}=0 \\
& f-\dot{x}=0
\end{aligned}
$$

Solving (4) with boundary conditions gives the optimal solution. Since this method is applied to agile airto-air missiles, assume that the initial conditions are given to every state. However, not all states have final conditions.

$$
\left\{\left\{x_{K}\right\},\left\{x_{F}\right\}\right\} \in x
$$

$x_{K} \in R^{n_{K}}$ and $x_{F} \in R^{n-n_{K}}$ denote states that final conditions are known and states that final conditions are free respectively. When final state is free, final costate condition, (6), needs to be satisfied from the optimal control theory.

$$
\lambda_{F}\left(t_{f}\right)=\frac{\partial \phi}{\partial x_{f}}
$$

where $\lambda_{F} \in R^{n-n_{K}}$ denotes costates related to states $x_{F}$. Note that if final state $x_{K f}$ is known, related costate's final value becomes free and when final state is free, related costate's final value has to meet (6). Moreover, all initial states are known whereas every initial costate is undetermined. Therefore, when initial costates are treated as unknown variables, solving optimal control problem becomes finding appropriate initial costates' value while final conditions are met. To illustrate mathematically, let $J_{2}$ be defined as:

$$
J_{2}=f\left(\lambda_{0}\right)=\sum_{i=1}^{n_{K}}\left(x_{K_{i}}\left(t_{f}\right)-x_{K_{i} f}\right)^{2}+\sum_{j=1}^{n-n_{K}}\left(\lambda_{F_{j}}\left(t_{f}\right)-\frac{\partial \phi}{\partial x_{F_{j} f}}\right)^{2}(7)
$$

The whole idea of parameterized costate optimization method is to make initial costates as parameters which are variables and find those values that satisfy $J_{2}=0$. In (7), the term $x_{K_{i}}\left(t_{f}\right)$ and $\lambda_{F_{j}}\left(t_{f}\right)$ come from the integration results of (4) with initial costate guess values. Therefore, note that $J_{2}$ is a function of initial costate values. It is important to make $J_{2}$ exactly zero so its solution is equivalent to the optimal solution.

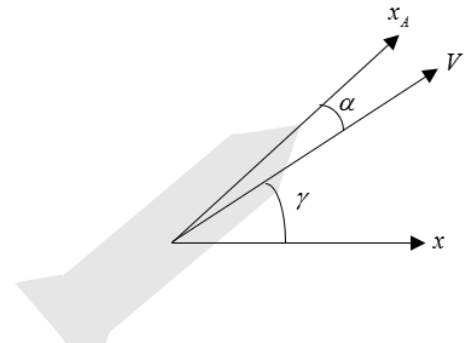

Figure 1. Simple missile model coordinate system.

\subsection{Simple missile model without control bound}

A simple missile model is employed in two dimensional yaw plane optimal control problem in order to verify pseudospectral method with parameterized costate optimization method.

The missile dynamics and optimal control problem are formulated as follows:

$$
\begin{gathered}
\dot{V}=a \cos \alpha \\
\dot{\gamma}=\frac{a \sin \alpha}{V} \\
J=\phi\left(x\left(t_{f}\right), t_{f}\right)+\int_{t_{0}}^{t_{f}} \mathrm{~g}(x(t), u(t), t) d t=-V_{f}
\end{gathered}
$$

$V, a, \alpha$, and $\gamma$ denote velocity, acceleration, angle of attack, and flight path angle respectively. The control command is $\alpha$. Since the agile turn problem is defined as maximizing terminal velocity, cost function is set to $-V_{f}$. Also initial and final conditions are:

$$
\begin{aligned}
& V(0)=V_{0} \\
& \gamma(0)=\gamma_{0}, \gamma\left(t_{f}\right)=\gamma_{t_{f}}
\end{aligned}
$$

Applying (4) and (6) to this problem derives

$$
\begin{array}{ll}
\dot{V}=a \cos \alpha & V(0)=V_{0}, V\left(t_{f}\right)=\text { free } \\
\dot{\gamma}=a \sin \alpha / V & \gamma(0)=\gamma_{0}, \gamma\left(t_{f}\right)=\gamma_{t_{f}} \\
\dot{\lambda}_{V}=\lambda_{\gamma} a \sin \alpha / V^{2} & \lambda_{V}(0)=\text { parameter }, \lambda_{V}\left(t_{f}\right)=-1 \\
\dot{\lambda}_{\gamma}=0 & \lambda_{\gamma}(0)=\text { parameter }, \lambda_{\gamma}\left(t_{f}\right)=\text { free }
\end{array}
$$

Such that

$$
\tan \alpha=\frac{\lambda_{\gamma}}{\lambda_{V} V}
$$

Finding $\lambda_{V 0}$ and $\lambda_{\gamma 0}$ that makes $J_{2}$ zero is equivalent to solving optimal control problem. $J_{2}$ is defined as:

$$
J_{2}=f\left(\lambda_{V 0}, \lambda_{\gamma 0}\right)=\left(\gamma\left(t_{f}\right)-\gamma_{t_{f}}\right)^{2}+\left(\lambda_{V}\left(t_{f}\right)+1\right)^{2}
$$


Following simulation results is conducted to compare the numerical solutions between using pseudospectral method and using parameterized costate optimization method.

Table 1. Simulation parameters

\begin{tabular}{|c|c|c|c|}
\hline \multicolumn{2}{|c|}{ Parameters } & Value & Unit \\
\hline$a$ & $\begin{array}{c}\text { Constant axial } \\
\text { acceleration }\end{array}$ & 350 & $\mathrm{~m} / \mathrm{s}^{2}$ \\
\hline$t_{f}$ & Boost time & 3 & $\mathrm{~s}$ \\
\hline$V_{0}$ & Initial velocity & 250 & $\mathrm{~m} / \mathrm{s}$ \\
\hline$\gamma_{0}$ & Initial flight path angle & 0 & $\mathrm{deg}$ \\
\hline$\gamma_{f}$ & Final flight path angle & 30 & $\mathrm{deg}$ \\
\hline
\end{tabular}

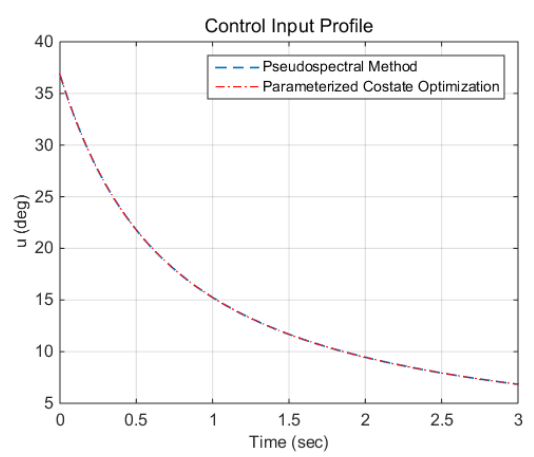

Figure 2. Control input $\alpha$ profile

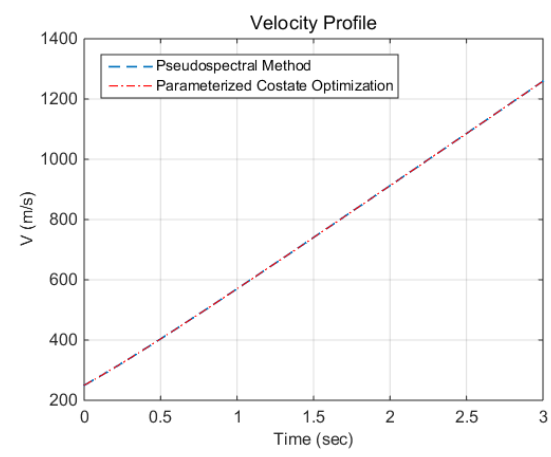

Figure 3. Velocity profile

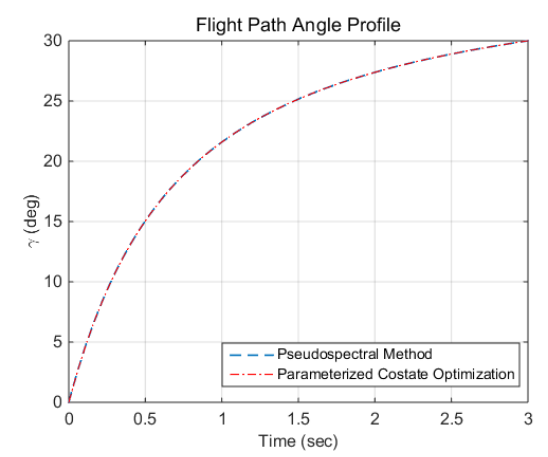

Figure 4. Flight path angle profile

The graphs in Fig. 2 and Fig. 3 show that both methods give the same results.

\subsection{Simple missile model with control bound}

Previous control input result shows that control input goes over 25 degrees which is too high for an actuator to follow that angle of attack command. Thus, control command needs to be bounded to a certain value which the actuator can withstand.

$$
|\alpha| \leq 25 \mathrm{deg}
$$

Same optimal control problem as in part 2.1 is treated in this control bounded problem.

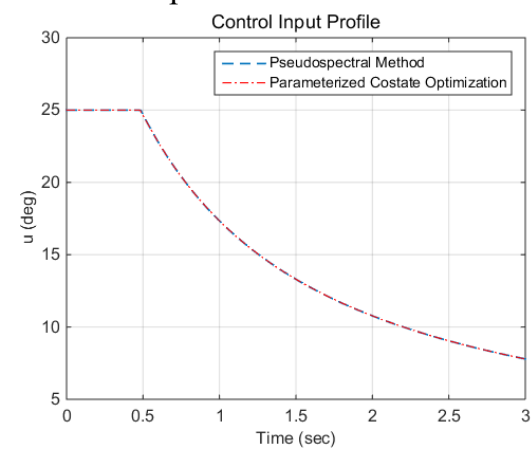

Figure 5. Control input $\alpha$ profile

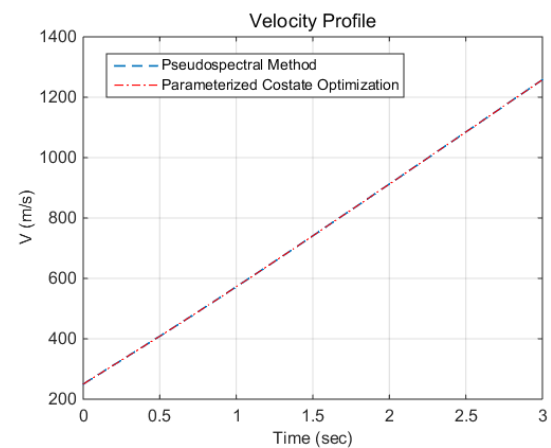

Figure 6. Velocity profile

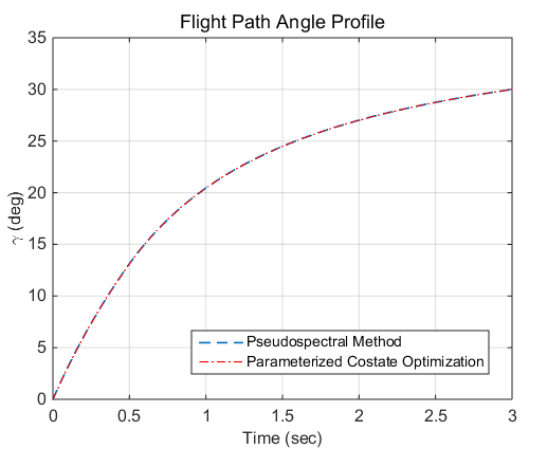

Figure 7. Flight path angle profile

When control is bounded, Pontryagin's minimum principle needs to be introduced. Fig. 4 shows bangsingular control. It starts with upper-bound bang control at first 0.5 seconds. This upper-bound bang control is determined from Pontryagin's minimum principle. However, after 0.5 seconds, it is following the singular arc which is determined from (14).

The parameterized costate optimization method verified pseudospectral method well on both unbounded control problem and bounded control problem.

\section{Terminal velocity maximization}


From the previous section, pseudospectral method is verified. Thus, pseudospectral method will be applied to terminal velocity maximization problem using the missile model with aerodynamic forces and thrust. This missile model considers mass variation $m(t)$; thrust $T(t)$; aerodynamic forces $L(\alpha, V)$ and $D(\alpha, V)$; and time lag $\tau$. Equations of motion are written as:

$$
\begin{gathered}
\dot{V}=\frac{T(t) \cos \alpha-D(\alpha, V)}{m(t)} \\
\dot{\gamma}=\frac{T(t) \sin \alpha+L(\alpha, V)}{m(t) V} \\
\dot{X}=V \cos \gamma \\
\dot{Y}=V \sin \gamma \\
\dot{\alpha}=\frac{u-\alpha}{\tau} \\
|u(t)| \leq 25 \mathrm{deg}
\end{gathered}
$$

Also, aerodynamic force models are given as Fig. 7. The lift and drag coefficients versus angle of attack are plotted at Mach number 0.7 to 1.0 with 0.1 increments.

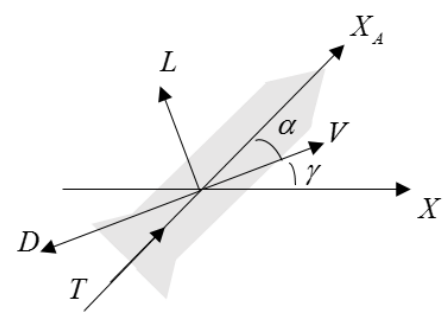

Figure 8. Missile Model Free Body Diagram

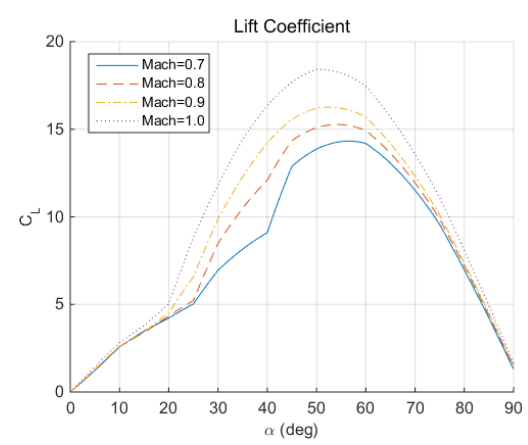

Figure 9. Lift Coefficients versus Angle of Attack

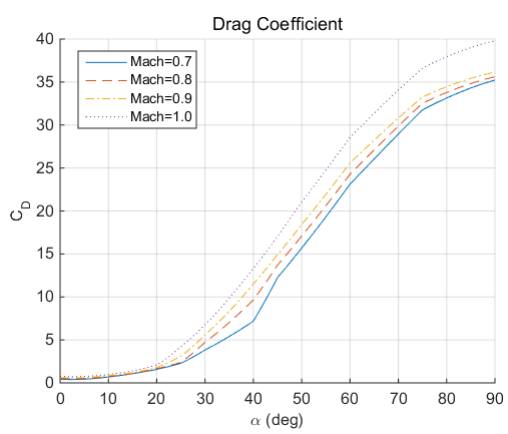

Figure 10. Drag coefficients versus angle of attack
Table 2. Missile Parameters

\begin{tabular}{|c|c|c|c|}
\hline \multicolumn{2}{|c|}{ Parameters } & Value & Unit \\
\hline$t_{f}$ & Boost time & 2.69 & $\mathrm{~s}$ \\
\hline$m_{0}$ & Initial mass & 92 & $\mathrm{~kg}$ \\
\hline$c$ & Mass burning rate & 13 & $\mathrm{~kg} / \mathrm{s}$ \\
\hline$T$ & Average thrust & 35000 & $\mathrm{~N}$ \\
\hline$S_{\text {ref }}$ & Reference wing area & 0.0182 & $\mathrm{~m}^{2}$ \\
\hline$\tau$ & Time constant & 0.3 & $\mathrm{~s}$ \\
\hline
\end{tabular}

Table 3. Simulation Parameters

\begin{tabular}{|c|c|c|c|}
\hline \multicolumn{2}{|c|}{ Parameters } & Value & Unit \\
\hline$\rho$ & Air density & 1.0065 & $\mathrm{~kg} / \mathrm{m}^{3}$ \\
\hline$\alpha_{0}$ & Initial angle of attack & 0 & $\mathrm{deg}$ \\
\hline$V_{0}$ & Initial velocity & 250 & $\mathrm{~m} / \mathrm{s}$ \\
\hline$\gamma_{0}$ & Initial flight path angle & 0 & $\mathrm{deg}$ \\
\hline$\gamma_{f}$ & Final flight path angle & $50,75,100$ & $\mathrm{deg}$ \\
\hline
\end{tabular}

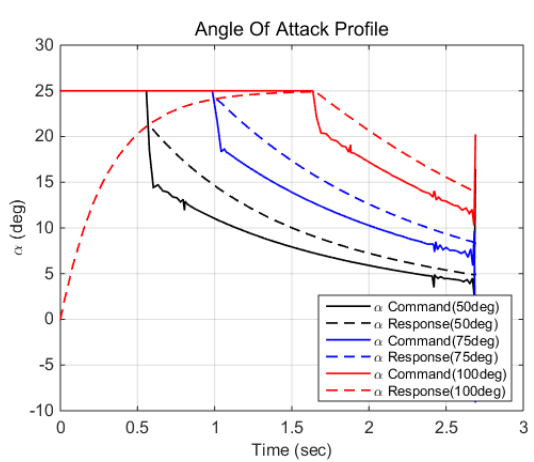

Figure 11. Angle of attack command and response profile

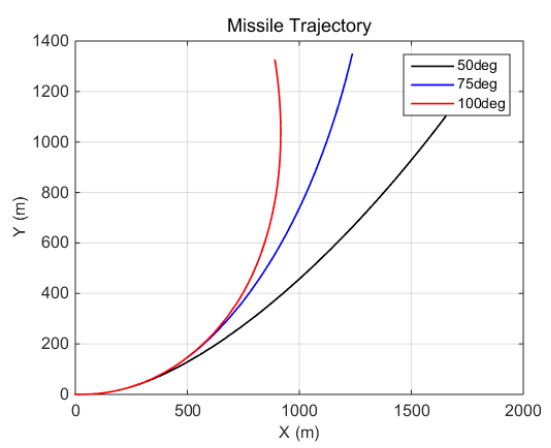

Figure 12. Missile trajectory

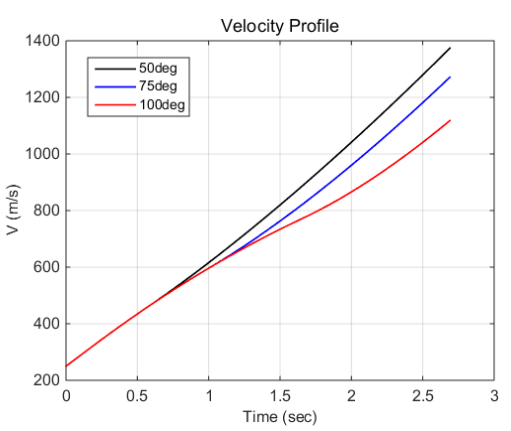

Figure 13. Velocity Profile 


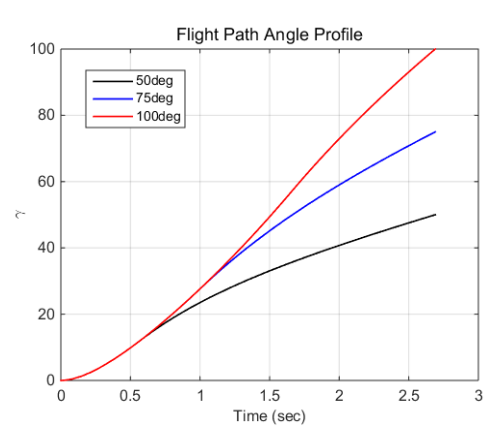

Figure 14. Flight Path Angle Profile

The missile parameters are from SRAAM model in [8]. Also MATLAB GPOPS-II software is applied to get the optimal solution of pseudospectral method. As the final flight path angle demands for bigger angles, velocity increase rate slows at the mid-time. This is because the agile air-to-air missiles use thrust more to the turning maneuver rather than increasing velocity.

The results of angle of attack command shown in Fig. 8 show that optimal control command follows bangsingular control. Since control is bounded at 25 degrees, control command remains at its maximum value for few seconds and then follows its singular arc. The bigger final flight path angle makes the bang control time longer. The bang-singular control could make velocity higher than bang-bang control since bang-bang control could lose missile's velocity when the second bang occurs.

\section{Conclusion}

Based on the analysis of the optimal control theory, parameterized costate optimization method is proposed as a numerical method of the optimal control problem. By applying this method, solutions obtained by pseudospectral method are verified. Those numerical methods were applied to air-to-air missiles' agile turn problem. The agile turn problem can be formulated as maximizing terminal velocity problem. Therefore, with simple missile model and missile model with aerodynamic forces and thrust, maximizing terminal velocity problem is studied. The optimal control was given as bang-singular control, which is a combination of bang-bang control and control that meets optimality necessary condition. For the future works, bang-singular control should be analyzed. This might include obtaining analytical solutions and finding bang off time.

\section{Acknowledgment}

The research is supported by LIG Nex1 (Y16-002).

\section{References}

1. K. A. Wise, and D. J. Broy, "Agile missile dynamics and control." Journal of Guidance, Control, and Dynamics, 21, pp. 441-449 (1998).

2. M. B. McFarland, and A. J. Calise, "Adaptive nonlinear control of agile antiair missiles using neural networks." Control Systems Technology, IEEE Transactions on, 8(5), pp. 749-756 (2000).

3. D. M. McDowell, G. W. Irwin, and G. McConnell, "Online neural control applied to a bank-to-turn missile autopilot." In Proceedings of the AIAA Guidance, Navigation, and Control Conference, pp. 1286-1294 (1995).

4. C. H. Lee, T. H. Kim, and M. J. Tahk, “Agile missile autopilot design using nonlinear backstepping control with time-delay adaptation." Transactions of the Japan Society for Aeronautical and Space Sciences, 57(1), pp. 9-20 (2014).

5. S. M. Ryu, D. Y. Won, C. H. Lee, and M. J. Tahk, "Missile autopilot design for agile turn control during boost-phase." International Journal Aeronautical and Space Sciences, 12(4), pp. 365-370 (2011).

6. D. Garg, M. Patterson, W. W. Hager, A. V. Rao, D. A. Benson, and G. T. Huntington, "A unified framework for the numerical solution of optimal control problems using pseudospectral methods." Automatica, 46(11), pp. 1843-1851 (2010).

7. M. A. Patterson, and A. V. Rao, "A General-Purpose MATLAB Toolbox for Solving Optimal Control Problems Using the Radau Pseudospectral Method." Manual for GPOPS-II, Gainesville, United States, pp. 2-3 (2013).

8. P. H. Zipfel, "Modeling and Simulation of Aerospace Vehicle Dynamics $2^{\text {nd }}$ edition" AIAA, Inc (2010). 